\title{
Prevalence and 7-year incidence of Type II diabetes mellitus in a Japanese-Brazilian population: an alarming public health problem
}

\author{
S. G. A. Gimeno ${ }^{1}$, S. R. G. Ferreira ${ }^{1}$, L. J. Franco ${ }^{2}$, A. T. Hirai ${ }^{1}$, L. Matsumura ${ }^{3}$, R. S. Moisés ${ }^{3 *}$ \\ ${ }^{1}$ Preventive Medicine Department, Federal University of São Paulo (UNIFESP-EPM), São Paulo, São Paulo, Brazil \\ 2 Preventive Medicine Department, Faculty of Medicine of Ribeirão Preto, São Paulo University (USP), Ribeirão Preto, São Paulo, Brazil \\ ${ }^{3}$ Internal Medicine Department, Federal University of São Paulo (UNIFESP-EPM), São Paulo, São Paulo, Brazil
}

\section{Abstract}

Aims/hypothesis. In 1993, the prevalence of glucose intolerance was studied in a sample of 647 firstgeneration and second-generation Japanese-Brazilians. Their cohort was followed until 2000, when a second survey was conducted, this included the first and second generations, aged 30 or more years. The aims were to estimate the prevalence of glucose intolerance and 7-yr incidence of Type II (non-insulindependent) diabetes mellitus in this population.

Methods. Prevalence rates were obtained for 1330 subjects examined in 2000. The incidence of diabetes mellitus was calculated for those classified as normal glucose tolerant in $1993(n=253)$. A Student's $t$ test and the Cox proportional hazard model were used in data analysis.

Results. In the year 2000, higher proportions of subjects were observed in all categories of glucose intolerance than those found in 1993. The overall inci- dence of diabetes was 30.9 per 1000 per year. A worse profile was observed among incident cases of diabetes, characterized by higher baseline values of anthropometric and metabolic variables as compared to those who had not developed diabetes. Analysis considering the simultaneous effects of demographic, nutritional and metabolic variables and physical activity levels for the development of diabetes showed that age, sex, waist circumference, fasting and 2-h plasma glucose concentrations were independent predictors.

Conclusion/interpretation. Our data point towards a worsening of glucose tolerance status among Japanese-Brazilians, who show one of the highest prevalence rates of diabetes mellitus worldwide. This could reflect their strong genetic susceptibility associated with unfavourable environmental conditions. [Diabetologia (2002) 45:1635-1638]

Keywords Incidence, prevalence, diabetes mellitus, risk factors, Japanese migrants.
A global epidemic of diabetes mellitus (DM) has been predicted. Studies [1] estimate that 250 million people will have diabetes by the year 2020, most of them will have Type II (non-insulin-dependent) diabetes mellitus. A strong genetic basis, environmental factors and

Received: 29 April 2002 / Revised: 2 July 2002

Published online: 24 October 2002

(C) Springer-Verlag 2002

*For The Japanese-Brazilians Diabetes Study Group: see Acknowledgements for list of group members

Corresponding author: Dr. S. G. A. Gimeno, Universidade Federal de São Paulo, Departamento de Medicina Preventiva, Rua Botucatu, 740, CEP 04023-062, São Paulo, SP, Brasil. E-mail: suely@medprev.epm.br. lifestyle changes have been implicated in the aetiology of Type II diabetes mellitus.

Despite the increasing prevalence of diabetes mellitus observed in Japan, which is among the top 10 countries estimating the number of adults with diabetes mellitus for the next years [1], its rate can be still considered relatively low. A prevalence of $7 \%$ and an incidence of 7.4 per 1000 per year were reported in Japanese people living in Japan [2]. We have reported the importance of diabetes mellitus, dyslipidemia and hypertension in Japanese-Brazilians [3,4] this is in agreement with studies conducted in migrants living in Washington, Hawaii and California [5].

This study estimated the prevalence of glucose intolerance and the 7-year incidence of Type II diabetes 
mellitus in a genetically homogeneous JapaneseBrazilian community and assessed the relation between some risk factors and the development of the disease.

\section{Subjects and methods}

In 1993, we started to follow up a sample of: (i) subjects born in Japan living in a Brazilian environment; (ii) subjects from first-generation Japanese parents. Both groups came from the Japanese-Brazilian community living in Bauru, a developed city of the São Paulo State, to help investigate the occurrence of glucose intolerance. In the first phase, 647 first-generation $(37.3 \%)$ and second-generation $(62.7 \%)$ Japanese-Brazilians aged 40 to 79 years were examined and submitted to an OGTT. Details on the selection and recruitment of the sample population have been previously described $[3,4]$. In the second phase of this study (1999-2000), in addition to the people enrolled in the first phase, the first-generation and second-generation Japanese-Brazilians aged 30 or more years $(n=1751)$ were invited to participate. Data were obtained from 1330 subjects; among 1104 new participants, there was $15.2 \%(n=168)$ of non-responders, including 23 deaths $(2.1 \%)$ which occurred during the collection period. There was a higher proportion of young $(<60 \mathrm{yrs})$ men among the non-examined subjects compared to the examined subjects. Among 647 participants of the first phase, the reasons for non-attendance $(39.0 \%)$ were death $(n=69)$, moving to other city or country $(n=57)$ and refusal $(n=127)$. Unimportant differences were observed concerning sex, age, generation and glucose tolerance status between nonparticipants and participants at baseline, in both phases of the study. From 394 subjects examined in 1993 and from 1999 to 2000, 141 subjects were excluded from incidence estimates due to the diagnosis of impaired fasting glycaemia (IFG), impaired glucose tolerance (IGT) or diabetes mellitus. Therefore, the incidence rate of diabetes mellitus was calculated for 253 subjects with normal glucose tolerance according to the 1993 GTT.

After written consent, subjects were interviewed using standardized questionnaires this included demographic, socio-economic, cultural, dietary aspects and physical activity information. Subjects were then scheduled for physical examination and laboratory procedures. Similar to the 1993 data collection, nutritional variables, blood pressure and metabolic profile were obtained in the 1999 to 2000 study. Blood pressure was measured by an automatic device (Omron model HEM-712C, Omron Health Care, USA). Fasting and 2-hr after 75 grams of oral glucose blood samples were obtained. The glucose load was administered to non-diabetic subjects and to those with self-reported diabetes mellitus but fasting capillary glucose of less than $11.1 \mathrm{mmol} / \mathrm{l}$, this was screened by glucose-oxidase strips. Plasma glucose was determined by the glucose-oxidase method. Glucose tolerance status was based on 1999 WHO criteria [6] or on the use of antidiabetic agents. Cholesterol contents of lipoprotein fractions and triglycerides were measured enzymatically.

Prevalence rates were estimated by point and by $95 \%$ confidence interval for the 1330 Japanese-Brazilians examined from 1999 to 2000. The rate of diabetes mellitus was calculated for 253 normal subjects in the 1993 OGTT. The Student's $t$ test was used to compare baseline parameters between diabetic patients and non-diabetic subjects. The Cox proportional hazard model was used to estimate crude and adjusted hazard ratios of demographic, metabolic and some lifestyle variables (total energy and macronutrient intakes and physical activity level).

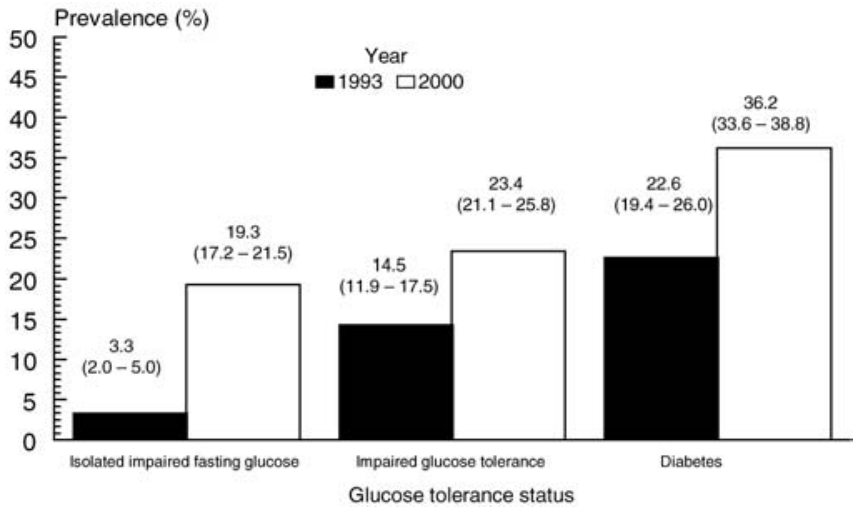

Fig. 1. Prevalence $(95 \% \mathrm{CI})$ of disturbances of glucose metabolism observed in 1993 and 2000 in Japanese-Brazilians. Standardized prevalence rates to age-distribution of world population in 1992: men: IFG: 4.4 vs $22.3 \%$; IGT: 11.4 vs $22.2 \%$; DM: 22.6 vs $39.6 \%$, in 1993 and in 2000, respectively; women: IFG: 2.1 vs $17.0 \%$; IGT: 16.4 vs $24.2 \%$; DM: 17.9 vs $30.7 \%$, in 1993 and in 2000, respectively

Statistical analysis was carried out using the Stata 7.0 software package (version 7.0 for WINDOWS; Stata Corporation, College Station, Tex., USA).

\section{Results}

In the year 2000, higher rates of disturbances of glucose metabolism were found compared to those observed in 1993 (Fig. 1). A greater increase was observed in the category of isolated IFG (approximately $550 \%$ ). From 1999 to 2000, 334 new cases of diabetes mellitus were identified among a total of 481 diabetic subjects. Similar to the 1993 study, a higher prevalence of diabetes mellitus $(p<0.05)$ was found in men as compared to women $(25.5 \%$ vs $19.9 \%$, in 1993 ; $40.6 \%$ vs $32.4 \%$, in 2000 ), but not concerning generation $(19.7 \%$ vs $24.4 \%$, in $1993 ; 39.3 \%$ vs $35.5 \%$, in 2000 for the first and second generations, respectively). In both phases, prevalence rates of IFG and IGT were higher among people aged 60 years or more compared to those less than 60 years of age $(47.0 \%$ vs $34.5 \%$, in $1993 ; 84.7 \%$ vs $74.5 \%$, in 2000 ). When an age of less than 40 years was considered, more than $50 \%$ showed some degree of glucose intolerance (IFG: 26.7\%; IGT: 14.7\%; DM: 15.5\%).

Until 2000, 51 of 253 normal subjects became diabetic, which resulted in an overall incidence of 30.9 per 1000 per year (95\% CI 23.5-40.6\% person-year), with 38.2 for men and 25.0 per 1000 per year for women (standardized incidence rates to age-distribution of world population: men 33.7 per 1000 personyear; women 21.1 per 1000 person-year).

The comparison of subjects' baseline characteristics showed a worse metabolic profile among incident cases of diabetes mellitus who had higher mean values of BMI, waist circumference, WHR, systolic blood 
Table 1. Hazard ratios (HR) and $95 \%$ CI for diabetes mellitus according to biological variables ${ }^{\mathrm{a}}$

\begin{tabular}{llll}
\hline & Crude HR & Adjusted HR & 95\% CI \\
\hline Sex (men vs women) & 1.52 & 1.95 & $1.04-3.65$ \\
Age (for each 10 yrs) & 0.77 & 0.65 & $0.80-0.90$ \\
Waist circumference (for each cm) & 1.05 & 1.05 & $1.01-1.09$ \\
Systolic blood pressure (for each mmHg) & 1.01 & 7.01 & $1.00-1.02$ \\
Fasting plasma glucose (for each mmol/l) & 6.99 & 1.33 & $3.32-14.77$ \\
2-hr plasma glucose (for each mmol/l) & 1.52 & $1.01-1.77$ \\
\hline
\end{tabular}

a Generation, BMI, WHR, triglycerides, HDL and family history of DM were excluded from the final model due to non-significant HR $(p>0.05)$

pressure, triglycerides, HDL, fasting and 2-h plasma glucose concentration in 1993 than the non-diabetic subjects in 2000. Also, newly diagnosed diabetic subjects were shown to have, in 1993, a higher mean daily intake of protein but not total energy, carbohydrate and fat intakes, when compared with those who had not developed the disease. No difference was observed concerning their physical activity level obtained in 1993 (data not shown).

Table 1 shows hazard ratios for diabetes mellitus, according to a number of variables at baseline (1993). Considering simultaneous effects of demographic, anthropometric, dietary and metabolic variables and activity level for the development of diabetes mellitus, sex, age, waist circumference, fasting and 2-h plasma glucose showed to be independent predictors of the disease.

\section{Discussion}

In this study, the high prevalence of glucose metabolism disturbances and incidence rates of diabetes mellitus found among Japanese-Brazilians suggest that an alarming epidemic of glucose intolerance is in course in this community. Despite limitations in comparing our findings with rates observed in other studies conducted in Asian [2] and non-Asian populations $[7,8]$, the rates of diabetes mellitus in JapaneseBrazilians are among the highest found worldwide. Our data indicated that the magnitude of the problem has worsened in the last 7 years. The profile of at-risk subjects to deteriorate glucose metabolism has remained the same, since diabetes mellitus is still more common among men and older subjects. However, the high prevalence of disturbances also among younger people aged 40 years or less $(56.9 \%)$ is remarkable. A projection raised from our data indicated that approximately 325 new cases of diabetes mellitus will be identified in the next five years among Japanese-Brazilians with IFG, IGT or normal glucose tolerance, if no preventative measure was instigated.

In this study, age, sex, waist circumference, fasting and 2-h plasma glucose were shown to be indepen- dent predictors for the development of diabetes mellitus. Despite the higher protein intake detected in the crude analysis among those who developed diabetes mellitus, a role for lifestyle variables could not be confirmed in multiple analysis. A higher number of men among those with newly diagnosed diabetes mellitus verified in our study is in agreement with some [7, 8] but not all studies [1]. A role for central obesity in the development of diabetes mellitus was expected. For the last decades, Pima Indians have represented the most important example of the increased risk of metabolic syndrome related to elevated adiposity [9]. Particularly, the importance of the central distribution of body fat has been emphasized. A substantial amount of evidence suggested that the combined analysis of BMI and fat distribution augments the predictive value for metabolic disturbances and cardiovascular disease. The combination of normal BMI and elevated WHR has been pointed out as one of the worst phenotypes to develop such abnormalities [10]. In agreement with our study, others preferred waist circumference as an indicative measure of visceral fat accumulation [11], which predisposes to insulin resistance and glucose intolerance. In fact, a 5\% increase in risk of diabetes mellitus for each centimetre of increase in waist circumference was detected.

This study addressed the overall unselected firstgeneration and second-generation Japanese-Brazilians living in a region of our country. Despite the possibility of bias generated by non-responders, we have no indications that this would change the dramatic picture of glucose intolerance seen in this population. Our assumption is based on the following aspects: (i) if among non-responders all subjects classified as normal glucose tolerant had been examined in the year $2000(n=101)$; (ii) if all of them had been exposed to development of diabetes mellitus for the mean time of observation (6.5 yrs); (iii) if no cases of diabetes mellitus had been identified among them, the incidence of diabetes mellitus would drop to 22.10 per 1000 per year, which still represents a markedly high rate. If we used a similar line of thought in relation to prevalence rates, the lowest prevalence expected for glucose metabolism abnormalities (IFG plus IGT plus 
DM) would be approximately $70 \%$. This shows that diabetes mellitus and its long-term complications should be considered as one of the major public health problems in this community.

Our data draws attention to the epidemic of glucose intolerance in Japanese migrants and their descendants in Brazil. Such an important public health problem could be occurring in other Japanese populations living outside Japan. This situation could be reflecting their strong genetic susceptibility associated with unfavourable environmental conditions related to the Western lifestyle.

Acknowledgements. We are indebted to the participants in the Japanese-Brazilians Diabetes Study for their continuing outstanding level of cooperation. This study was supported by a grant from the Fundação de Amparo à Pesquisa do Estado de São Paulo. Members of the Japanese-Brazilians Diabetes Study Group: Alcides Hirai, MD; Amélia T. Hirai, MD; Helena Harima, MD; Katsumi Osiro MD; Magid Iunes, $\mathrm{MD}, \mathrm{PhD}$ (in memoriam); Mário Kikuchi PhD; Sandra R. G. Ferreira, MD, PhD; Suely G. A. Gimeno, PhD (Preventive Medicine Department, Federal University of São Paulo, Brazil); Laércio J. Franco, MD, PhD (Preventive Medicine Department, Faculty of Medicine of Ribeirão Preto, São Paulo University, Brazil); Luiza Matsumura, MD, PhD; Regina S. Moisés, MD, PhD (Internal Medicine Department, Federal University of São Paulo, Brazil); Marly A. Cardoso, PhD (Nutrition Department, Faculty of Public Health, São Paulo University, Brazil); Newton de Barros Jr., MD, PhD (Surgery Department, Federal University of São Paulo, Brazil); Nilce Tomita, PhD (Faculty of Odontology of Bauru, São Paulo University, Brazil); Katsunori Wakisaka (Japanese-Brazilians Study Center, Brazil); and Rita Chaim (Nutrition Department, Sagrado Coração de Jesus University, Bauru, Brazil).

\section{References}

1. King H, Aubert RE, Herman WH (1998) Global burden of diabetes, 1995-2025: prevalence, numeric estimates and projections. Diabetes Care 21:1414-1431

2. Tsumura K, Hayashi T, Suematsu C, Endo G, Fujii S, Okada K (1999) Daily alcohol consumption and the risk of type 2 diabetes in Japanese men: the Osaka Health Survey. Diabetes Care 22:1432-1437

3. Ferreira SRG, Iunes M, Franco LJ et al. (1996) Disturbances of glucose and lipid metabolism in first and second generation Japanese-Brazilians. Diabetes Res Clin Pract 34:59-63

4. Ferreira SRG, Franco LJ, Gimeno SGA, Iochida LC, Iunes M (1997) Is insulin or its precursor independently associated with hypertension? An epidemiological study in Japanese-Brazilians. Hypertension 30:641-645

5. Fujimoto WY, Leonetti DL, Kinyoun JL et al. (1987) Prevalence of diabetes mellitus and impaired glucose tolerance among second generation Japanese-American men. Diabetes 36:730-738

6. Alberti KGMM, Zimmet PZ for the World Health Organization Consulation (1999) Definition, diagnosis and classification of diabetes mellitus and its complications. Part 1: Diagnosis and classification of diabetes mellitus. Report of a WHO Consulation WHO, Geneva

7. Vilbergsson S, Sigurdsson G, Sigvaldason H et al. (1997) Prevalence and incidence of NIDDM in Iceland: evidence for stable incidence among males and females 1967-1991 - The Reykjavik Study. Diabet Med 14:491-498

8. Berger B, Stenstrom G, Sundkvist G (1999) Incidence, prevalence and mortality of diabetes in a large population: a report from the Skaraborg Diabetes Registry. Diabetes Care 22:773-778

9. Knowler WC, Pettitt DJ, Saad MF et al. (1991) Obesity in the Pima Indians: its magnitude and relationship with diabetes. Am J Clin Nutr 53:1543S-1551S

10. Samaras K, Campbell LV (2000) Increasing incidence of type 2 diabetes in the third millennium: is abdominal fat the central issue? Diabetes Care 23:441-442

11. Inoue S, Zimmet P, Caterson I et al. (2000) WHO Western Pacific Region. The Asia-Pacific perspective: redefining obesity and its treatment. Health Communications Australia 